\title{
Interactions Between Positronium Atoms in Porous Silica
}

\author{
D. B. Cassidy and A.P. Mills, Jr. \\ Department of Physics and Astronomy, University of California, Riverside, California 92521-0413, USA
}

(Received 7 September 2007; published 7 January 2008)

\begin{abstract}
Interactions between pairs of positronium (Ps) atoms confined in porous silica films have been directly observed for the first time. Because of selection rules, the nature of such interactions should depend on the structure of the porous medium: if a Ps surface state exists, dipositronium $\left(\mathrm{Ps}_{2}\right)$ molecules may be created, and if there is a continuum of cavity energy levels, spin exchanging collisions may occur. Using two structurally different silica films, we have been able to isolate and study these two processes. Our data indicate that $\mathrm{Ps}_{2}$ formation occurs primarily via a Langmuir-Hinshelwood-type mechanism on the internal pore surfaces, with an interaction length of the order of $7 \times 10^{-8} \mathrm{~cm}$, and that the effective cross section for nonthermalized Ps-Ps spin exchange quenching in porous silica is around $9 \times 10^{-15} \mathrm{~cm}^{2}$.
\end{abstract}

DOI: 10.1103/PhysRevLett.100.013401

PACS numbers: 36.10.Dr, 34.80.Nz, 82.33.Ln

It has recently become possible to study interactions between positronium (Ps) atoms by implanting intense pulses of positrons into porous silica films [1]. This leads to the formation of Ps atoms that may become trapped in the internal voids $[2,3]$ with a significant probability of overlapping. Specifically, if two oppositely polarized triplet atoms collide, they may join together to form a $\mathrm{Ps}_{2}$ molecule $[4,5]$ or exchange spins and both be converted into the short lived singlet state [6] or the $m=0$ triplet state. Since the $\mathrm{Ps}_{2}$ molecule decays rapidly, with a lifetime of $\sim 0.25 \mathrm{~ns}$ [7], and the high magnetic field used in the experiment quenches the $m=0$ triplet atoms [8], all of these mechanisms increase the mean Ps decay rate; we refer to them generally as "quenching" and to those that involve Ps atoms changing their spin states as spin exchange quenching (SEQ).

In our previous experiments [1], the time resolution of the system was insufficient to observe quenching effects directly, and the occurrence of such was of necessity inferred from the latter parts of our lifetime spectra. In this Letter, we describe experiments performed using an upgraded positron beam [9] and a faster detector [10], which have allowed us to observe directly the density dependent change in the Ps decay rate. Furthermore, by using silica films with different structural properties, and by varying their temperature, we have been able to distinguish between the $\mathrm{Ps}_{2}$ and SEQ mechanisms.

The experimental arrangement and methodologies have been described in detail elsewhere [9]. Briefly, sub-ns positron pulses of varying areal densities were implanted into the target films in a strong $(\sim 1.5 \mathrm{~T})$ magnetic field, and the resulting annihilation radiation was detected by a $\mathrm{PbF}_{2}$ Cherenkov radiator attached to a photomultiplier (PMT) [10] from which single-shot lifetime spectra [11] were obtained.

Two different silica films were used which, quite fortuitously, had the complimentary properties necessary to isolate $\mathrm{Ps}_{2}$ and SEQ events. The first target $(S 1)$ was a $45 \%$ porous silica film, $230 \mathrm{~nm}$ thick with a $50 \mathrm{~nm}$ non- porous capping layer. This film contained randomly aligned, interconnected pores with a diameter of $4 \mathrm{~nm}$ [12]. The second target (S2) was $450 \mathrm{~nm}$ thick with a pore diameter of $3 \mathrm{~nm}$ and a porosity of $\sim 70 \%$. In this sample, the pores were aligned and interconnected along one dimension, making a set of (roughly) cylindrical tubular channels parallel to the film surface [13].

Single shot lifetime spectra (averaged over 12 shots) taken with both samples at high and low beam densities are shown in Fig. 1(a). Figures 1(b) and 1(c) show the differences between the high and low density data. Since

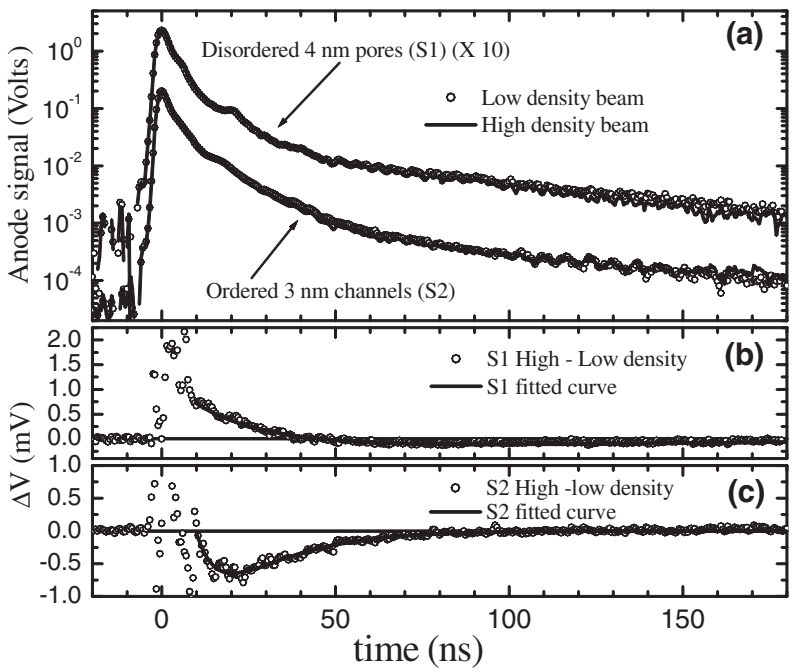

FIG. 1. (a) Lifetime spectra at room temperature using samples $S 1$ and $S 2$ taken at low $\left(S 1=9.5 \times 10^{9} \mathrm{~cm}^{-2}, S 2=\right.$ $\left.6.7 \times 10^{9} \mathrm{~cm}^{-2}\right)$ and high $\left(S 1=2.9 \times 10^{10} \mathrm{~cm}^{-2}, S 2=2.5 \times\right.$ $10^{10} \mathrm{~cm}^{-2}$ ) beam areal densities. The $S 1$ data are displaced (by a factor of 10) for clarity. The bump in the curves at about $19 \mathrm{~ns}$ is due to light feedback in the PMT, and smaller wiggles at later times are caused by internal cable reflections. $(b-c)$ Difference between the high and low density data for $S 1$ and $S 2$, respectively, and the fits (as described in the text) from which the parameters $\beta_{S 1}$ and $\beta_{S 2}$ were obtained. 
only features that depend on the beam density are present in the difference curves, these data constitute a direct observation of the quenching process.

Ps localized in a porous material decays primarily via interactions with the surrounding medium, and hence the decay rate depends on the pore size [3,14]; if Ps atoms are able to interact with each other, however, there will be an additional component to the decay. In this case, the number of Ps atoms, $n$, at time $t$ will be described by a (nonlinear) differential equation:

$$
d n(t, \beta) / d t=-\gamma n(1+\beta n) .
$$

Here, $\gamma$ is the linear Ps decay rate (due to self annihilation and/or interactions with the surrounding medium), and $\beta$ is a parameter that describes the nonlinear, densitydependent decay due to Ps-Ps interactions. $\beta$ is defined using a normalization such that $n(t=0)=1$. The difference between the high and low density spectra, $\Delta V$ [see Figs. 1(b) and 1(c)], may be written as

$$
\begin{aligned}
\Delta V & \propto d n(t, \beta) / d t-d n(t, 0) / d t \\
& =A \gamma\left(\exp (-\gamma t)-\frac{(1+\beta) \exp (\gamma t)}{[(1+\beta) \exp (\gamma t)-\beta]^{2}}\right),
\end{aligned}
$$

where A is an arbitrary scaling constant and it is assumed that there are no Ps-Ps interactions $(\beta=0)$ when a low density pulse is used. Fitting Eq. (2) to the data of Figs. 1(b) and 1(c), we obtain $\beta_{S 1}=1.35 \pm 0.06, \gamma_{S 1}=$ $0.011 \pm 0.05 \mathrm{~ns}^{-1}, \quad$ and $\quad \beta_{S 2}=0.95 \pm 0.11, \quad \gamma_{S 2}=$ $0.023 \pm 0.001 \mathrm{~ns}^{-1}$, respectively. We truncate our fits at time $t_{0}=10 \mathrm{~ns}$ because the finite resolution and noise in the prompt peak make the data unreliable at very early times.

As we shall explain, we interpret the quenching in $S 1$ as being due solely to the formation of $\mathrm{Ps}_{2}$ molecules, and that in $S 2$ as being due only to SEQ. When Ps is initially emitted into the voids (of both samples), it has an energy of around $1 \mathrm{eV}$ [15] and can take 10's of ns to thermalize [16], depending on the condition of the pore surfaces [17]. The quenching in both $S 1$ and $S 2$ is rapid, however, and it is likely that the Ps in $S 2$ is not thermal. Conversely, in $S 1$, molecule formation occurs via surface states, so these atoms must be thermalized (a hypothesis which is confirmed by the thermal desorption data shown below). This implies that some fraction of the Ps atoms in $S 1$ are "born" in a surface state. In $S 2$, where there is no surface state, the Ps atoms are probably thermalizing throughout the quenching process, which complicates our determination of the SEQ cross section; this is something we should like to know, insofar as it determines the rate at which a partially spin polarized ensemble of Ps atoms will become fully spin polarized, an important consideration in experiments to create a Ps Bose-Einstein condensate (BEC) [18].

We shall approximate SEQ as a free particle scattering event, and estimate the cross section, $\sigma_{\mathrm{SEQ}}$, in the following way: At $t=0$, the decay rate due to spin exchange between approximately equal numbers of $m=+1$ and $m=-1 \mathrm{o}-\mathrm{Ps}$ atoms is

$$
\beta \gamma=\sqrt{2}\langle n\rangle \sigma_{\mathrm{SEQ}} \bar{v} .
$$

Here, $\bar{v}$ is the mean Ps speed, and $\langle n\rangle$ is the mean density of $m=+1$ Ps atoms. We estimate a mean Ps energy of $0.25 \mathrm{eV}$ during the quenching [19], so that $\bar{v} \approx$ $2 \times 10^{7} \mathrm{~cm} / \mathrm{s}$. We approximate the mean Ps density as $\langle n\rangle=f_{m=1} N_{p} / p w^{2} z_{1 / 2}$, where $f_{m=1}=0.013$ is the fraction of positrons that form Ps in the $m=1$ (or -1 ) state, $N_{p}$ is the number of positrons per pulse $\left(1.8 \times 10^{7}\right), p$ is the sample porosity $(0.7), w$ is the full width at half maximum (FWHM) of the Gaussian areal beam profile $(250 \mu \mathrm{m})$ [9], and $z_{1 / 2}$ is the mean positron implantation depth $(60 \mathrm{~nm})$ [20]. In this way, we find $\langle n\rangle=$ $8.9 \times 10^{13} \mathrm{~cm}^{-3}$, and then $\sigma_{\mathrm{SEQ}} \approx 9 \times 10^{-15} \mathrm{~cm}^{2}$. Owing to the uncertainties in the Ps density and thermalization rate, we estimate that this is probably only accurate to, at best, a factor of 2 .

The cross section we measure is an effective cross section describing two distinct processes. That is,

$$
\sigma_{\mathrm{SEQ}}=\sigma_{1-1,11 \rightarrow 00,00}+\sigma_{1-1,11 \rightarrow 10,10} .
$$

Here we have used the same notation as that employed by Ivanov, Mitroy, and Varga [21] in which the cross sections are labeled according to the total spin $s$ and the spin projection $m$ of the incoming and outgoing Ps states thusly, $\sigma_{s_{1} m_{1}, s_{2} m_{2} \rightarrow s_{1}^{\prime} m_{1}^{\prime} s_{2}^{\prime} m_{2}^{\prime}}$. The first term on the right of Eqn. (4) describes the spin exchanging reaction in which an $m=1$ triplet atom colliding with an $m=-1$ triplet atom leads to two singlet atoms, and the second term refers to an interaction in which the outgoing atoms are both $m=0$ triplets; the latter atoms are rapidly quenched in the magnetic field, and both processes are essentially indistinguishable in the present experimental configuration.

The calculated value for $\sigma_{\mathrm{SEQ}}$ as defined above is $5.2 \times$ $10^{-15} \mathrm{~cm}^{2}$ in the zero energy limit, while at $0.25 \mathrm{eV}$ it is $\sim 2.1 \times 10^{-15} \mathrm{~cm}^{2}$ ) [21]. Our measurement of $\sigma_{\mathrm{SEQ}}$ is therefore consistent with the theoretical value, albeit with a rather large error.

A similar analysis of the data obtained with sample $S 1$ yields an interaction length $R$ for Ps atoms on the pore surfaces of $R \sim 7 \times 10^{-8} \mathrm{~cm}$. This determination does not take into account the correct surface structure and should be considered an order of magnitude estimate only.

One of the most significant differences between the two samples used is the fact that $S 1$ appears to have a Ps surface state while $S 2$ does not. The presence of such a surface state is indicated by an increase in the amount of long-lived Ps present when the sample is heated, due to the thermal desorption of surface atoms [22]. This is indicated in Fig. 2 which shows the parameter $f_{d}$ as a function of temperature for the two samples. This parameter is the integral of lifetime spectra from 20 to $150 \mathrm{~ns}$ divided by the integral from -20 to $150 \mathrm{~ns}$, and is a measure of the amount of 


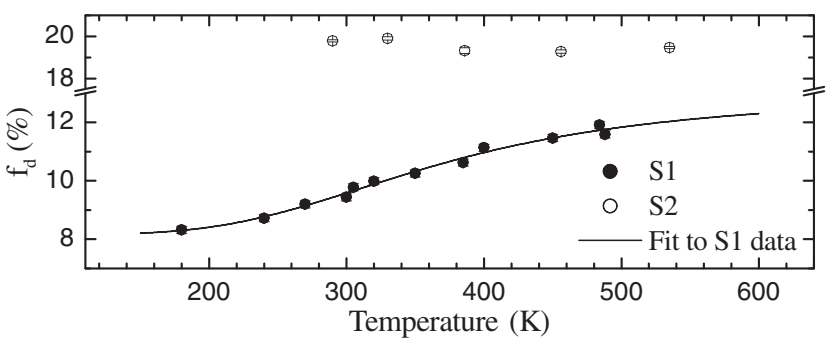

FIG. 2. $f_{d}$ as a function of temperature for $S 1$ (filled circles) and $S 2$ (open circles) taken at the low beam density. The $S 2$ data were taken using a reduced magnetic field of $0.15 \mathrm{~T}$ to minimize the effect of radiation damage, which leads to an increase in $f_{d}$. A fit was made to the $S 1$ data using the procedure described in the text.

(long-lived) Ps present. It is evident from Fig. 2 that $f_{d}$ increases with temperature for $S 1$ but not for $S 2$. It is not clear why $S 1$ has a Ps surface state and $S 2$ does not [23].

$f_{d}$ was measured as a function of the beam density $n_{2 D}$ for both samples. To reduce the effects of drift, the density was cycled repeatedly through five (or six) values from high to low density. The mean value of the 5 (6) $f_{d}$ measurements in each cycle was subtracted from the 5 (6) measurements in the cycle, and the corrected measurements were averaged over all the cycles to obtain $\Delta f_{d}$ as a function of density, as shown in Fig. 3. This figure shows measurements made at different sample temperatures, and it is clear that the amount of quenching (characterized by the slope $\left.Q=d \Delta f_{d} / d n_{2 D}\right)$ in $S 1$ is reduced as the temperature is increased, while the quenching in $S 2$ does not depend significantly on the temperature. We interpret this as being due to the formation of $\mathrm{Ps}_{2}$ molecules by Ps atoms

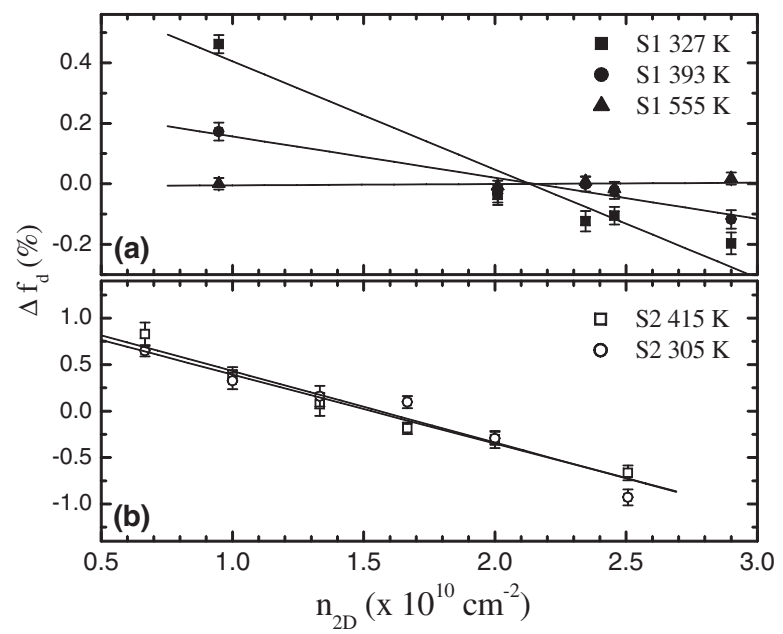

FIG. 3. The density dependence of $\Delta f_{d}$ for the $S 2$ (a) and $S 1$ (b) samples for various temperatures, as indicated in the legends. The solid lines are linear fits to the data which determine the parameter $Q$ (described in the text). We note that the magnitude of the quenching signal seen in $S 2\left(Q \sim 75 \times 10^{-14} \mathrm{~cm}^{-2}\right)$ is a lower limit as the sample had been partially damaged by irradiation with the positron beam. in the surface state in $S 1$ [5]. When all of the Ps atoms have been desorbed from the surface state, $\mathrm{Ps}_{2}$ formation cannot occur, but the desorbed atoms are prevented from taking part in spin exchanging collisions because the discrete cavity energy levels cannot accommodate the hyperfine energy difference between the incoming and outgoing Ps states, so at high temperatures, there is no quenching at all. We hypothesize there is no such suppression of SEQ in $S 2$ because the pores are aligned and the energy levels are not discrete. Since there is no surface state in $S 2, \mathrm{Ps}_{2}$ formation is unlikely to occur therein.

Figure 4 shows the temperature dependence of the quenching signal $Q(T)$ in $S 1$, which is parameterized by the slope of data such as that shown in Fig. 3(a). In the absence of three body collisions, $\mathrm{Ps}_{2}$ formation may occur via two different mechanisms: a reaction between two Ps atoms in a surface state (known as a LangmuirHinshelwood (LH) reaction [24]) or between a surface state and a "gas phase" Ps atom (known as an EleyRideal (ER) reaction [25]). Figure 2 shows a fit to the positronium fraction as a function of temperature, which provides the normalized thermal positronium yield $Y(T)$, such that $0 \leq Y(T) \leq 1$ [26]. Since the formation of $\mathrm{Ps}_{2}$ depends on the population of the surface state, we would expect a quenching signal resulting from an ER process to have the form $Q(T) \propto Z$, where $Z \equiv 1-Y(T)$, since $Y(T)$ refers to single atom desorption. If two surface atoms are involved in the quenching (LH model), then we should expect $Q(T) \propto Z^{2}$. In Fig. 4, both of these functions (scaled to match the low temperature value of $Q(T)$ ) are plotted along with the measured quenching data [5]. The LH model is clearly a better match to the data (note: this is not a fit) indicating that this mechanism is most likely the predominant mode of $\mathrm{Ps}_{2}$ formation [27].

In our prior work [1], we argued that the apparent density dependent change in the Ps decay rate could not be due to the inhibition of Ps formation by spur electrons, and the present data show conclusively that this is indeed the case; the quenching has been directly observed (Fig. 1).

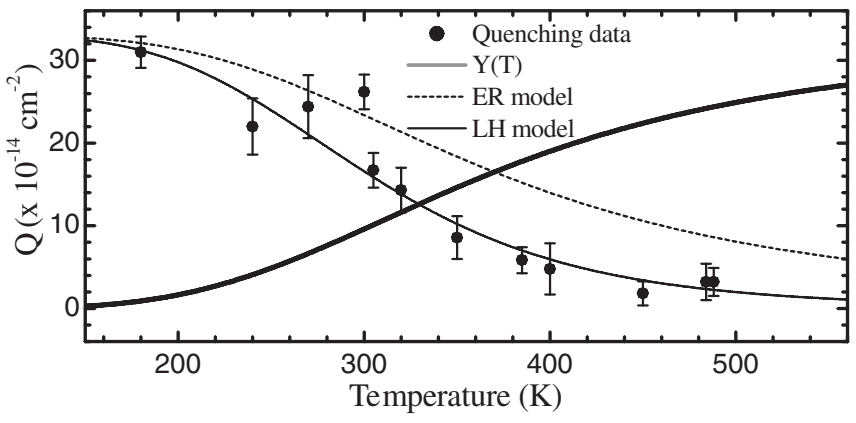

FIG. 4. The quenching in sample $S 1$ as a function of the temperature. The thermal positronium yield $Y(T)$ was obtained from a fit to the data shown in Fig. 3 and has been scaled to fit the amplitude of the quenching signal. The expected ER and LH model curves are plotted as described in the text. 
In light of the observed temperature dependence of the quenching, however, our previous speculation that our data might be due to a combination of $\mathrm{Ps}_{2}$ formation and SEQ is shown to be incorrect. In that work, a sample very similar to $S 2$ was used, and it is therefore unlikely that any $\mathrm{Ps}_{2}$ formation occurred. Moreover, our estimate of the SEQ cross section did not take into account the slow Ps thermalization, and, in the absence of a direct measurement of the quenching rate, relied on an oversimplified model. The present determination, while itself somewhat imprecise, supersedes the former. Given the complex nature of the SEQ process under these experimental conditions, a Monte Carlo simulation is probably required to properly account for the inhomogeneous Ps density distribution and the fact that the Ps atoms are not thermal.

To summarize, by using sub-ns positron pulses and an improved detection system, we have been able to directly observe Ps-Ps interactions in porous silica films. Varying the sample temperature and using different types of films have made it possible to distinguish between and study $\mathrm{Ps}_{2}$ and SEQ effects. Our data are consistent with $\mathrm{Ps}_{2}$ formation occurring via an LH type mechanism, in accordance with a recent estimate for quartz surfaces [28], which suggests that this might be a good material for future laser spectroscopy studies of molecular positronium in vacuum. Our estimate of the SEQ cross section is broadly consistent with the calculation by Ivanov, Mitroy, and Varga [21], and reiterates the need to use a spin polarized positron beam in future experiments to create a Ps BEC.

We are pleased to acknowledge, M. W. J. Bromley, R. G. Greaves, and J. Mitroy for helpful discussions and T. Maruo, N. Nishiyama, and H. K. M. Tanaka for providing the porous silica samples. This work was supported in part by the National Science Foundation under Grants Nos. PHY 0140382, PHY 0537431, PHY 0555701, and DMR 0216927.

[1] D. B. Cassidy, S. H. M. Deng, R. G. Greaves, T. Maruo, N. Nishiyama, J. B. Snyder, H. K. M. Tanaka, and A. P. Mills, Phys. Rev. Lett. 95, 195006 (2005).

[2] W. Brandt, S. Berko, and W. W. Walker, Phys. Rev. 120, 1289 (1960).

[3] D. W. Gidley et al., Phys. Rev. B 60, R5157 (1999).

[4] J. A. Wheeler, Ann. N.Y. Acad. Sci. 48, 219 (1946).

[5] D. B. Cassidy and A.P. Mills, Jr., Nature (London) 449, 195 (2007).

[6] M. Deutsch, Phys. Rev. 82, 455 (1951).

[7] D. M. Schrader, Phys. Rev. Lett. 92, 043401 (2004).

[8] A. Rich, Rev. Mod. Phys. 53, 127 (1981).

[9] D. B. Cassidy, S.H.M. Deng, R. G. Greaves, and A.P. Mills, Jr., Rev. Sci. Instrum. 77, 073106 (2006).

[10] D. B. Cassidy and A. P. Mills, Jr., Nucl. Instrum. Methods Phys. Res., Sect. A 580, 1338 (2007).

[11] D. B. Cassidy, S. H. M. Deng, H. K. M. Tanaka, and A.P. Mills, Jr., Appl. Phys. Lett. 88, 194105 (2006).
[12] More details regarding sample $S 1$ can be found in H. K. M. Tanaka, T. Kurihara, and A. P. Mills, Jr., Phys. Rev. B 72, 193408 (2005).

[13] More details regarding samples like $S 2$ can be found in S. Tanaka, N. Nishiyama, Y. Oku, Y. Egashira, and K. Ueyama, J. Am. Chem. Soc. 126, 4854 (2004).

[14] S. J. Tau, J. Chem. Phys. 56, 5499 (1972); M. Eldrup, D. Lighbody, and J. N. Sherwood, Chem. Phys. 63, 51 (1981).

[15] Y. Nagashima, Y. Morinaka, T. Kurihara, Y. Nagai, T. Hyodo, T. Shidara, and K. Nakahara, Phys. Rev. B 58, 12676 (1998).

[16] Y. Nagashima, M. Kakimoto, T. Hyodo, K. Fujiwara, A. Ichimura, T. Chang, J. Deng, T. Akahane, T. Chiba, K. Suzuki, B. T. A. McKee, and A. T. Stewart, Phys. Rev. A 52, 258 (1995).

[17] C. He et al., Phys. Rev. B 75, 195404 (2007).

[18] P. M. Platzman and A. P. Mills, Jr., Phys. Rev. B 49, 454 (1994); D. B. Cassidy and A. P. Mills, Jr., Phys. Status Solidi C 4, 3419 (2007).

[19] This estimate is based on thermalization data in pores similar to those used here. See, H. Saito and T. Hyodo in New Directions in Antimatter Chemistry and Physics, edited by C. M. Surko and F.A. Gianturco (Kluwer, Dordrecht, 2001), p. 101.

[20] This parameter was calculated using a mean positron beam energy of $1.5 \mathrm{keV}$. J. Algers, P. Sperr, W. Egger, G. Kögel, and F. H. J. Maurer, Phys. Rev. B 67, 125404 (2003).

[21] I. A. Ivanov, J. Mitroy, and K. Varga, Phys. Rev. A 65, 022704 (2002).

[22] P. Sferlazzo, S. Berko and K. F. Canter, Phys. Rev. B 32, 6067 (1985).

[23] Another difference between the two samples is that $S 2$ exhibited significant radiation damage following exposure to the positron beam. This limited the amount of data that could be acquired in a single run, but did not otherwise affect the experiments described here. Details are given in D. B. Cassidy and A. P. Mills, Jr., Nucl. Instrum. Methods Phys. Res., Sect. B 262, 59 (2007).

[24] E.g., S. Morisset, F. Aguillon, M. Sizun, and V. Sidis, J. Chem. Phys. 122, 194702 (2005).

[25] E.g., J. G. Quattrucci and B. Jackson, J. Chem. Phys. 122, 074705 (2005).

[26] As explained in Ref. [5], the fit was made to the function $Y(T)=\left[1+(300 \mathrm{~K} / T)^{2} S^{-1}\left(7.2 \times 10^{-7}\right) \exp \left\{E_{a} / k T\right\}\right]^{-1}$ where $E_{a}$ is the activation energy and $S$ is the sticking coefficient of the surface Ps atoms.

[27] As described in Ref. [5], one may fit the quenching data to a function proportional to $Z$ using a sticking coefficient that is approximately 3 times larger. Since the thermalization rate of Ps may be affected by the presence of low mass surface atoms (Ref. [17]), one could speculate that the presence of a Ps atom on a surface might increase the sticking coefficient of a second atom by providing a more efficient energy loss mechanism. In this case, the quenching could have a temperature dependence governed by single Ps atom desorption, even if the $\mathrm{Ps}_{2}$ were formed via an LH mechanism.

[28] R. Saniz, B. Barbiellini, P. M. Platzman, and A. J. Freeman, Phys. Rev. Lett. 99, 096101 (2007). 